\begin{tabular}{|c|c|c|}
\hline 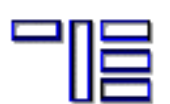 & $\begin{array}{l}\text { Revista Electrónica } \\
\text { Educación y Cultura en }\end{array}$ & $\begin{array}{l}\text { Educación. } \\
\text { la Información. }\end{array}$ \\
\hline & http://www.usal.es/teoriaeducacion & Vol. 9. $N^{\circ}$ 3. Noviembre 2008 \\
\hline
\end{tabular}

\title{
UNA APROXIMACIÓN A LA VIRTUALIDAD EDUCATIVA DE LOS VIDEO- JUEGOS
}

Resumen. ¿Es posible que una plataforma que exige la continua participación del usuario pueda afectar a su actitud ante la participación entendida de un modo más amplio? ¿Se pueden adquirir actitudes y habilidades sociales y participativas a través de los videojuegos?

Ninguna de estas preguntas tiene una respuesta sencilla; a lo largo de este artículo se plantean y discuten varios puntos de reflexión que tratan de darles respuesta en el marco de la concreta realidad que rodea a los adolescentes

Palabras clave: Juego, videojuegos, participación, habilidades y actitudes sociales, adolescentes.

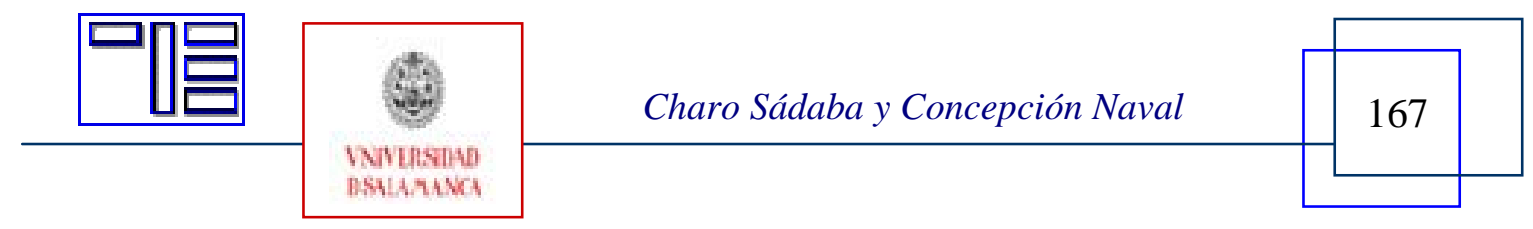


Revista Electrónica Teoría de la Educación.

Educación y Cultura en la Sociedad de la Información.

http://www.usal.es/teoriaeducacion

Vol. 9. No 3. Noviembre 2008

\section{AN APPROACH TO THE VIRTUAL EDUCATION OF VIDEO-GAMES}

Abstract. Could a platform, as videogames, that requires the active participation of users, be helpful to develop a broader participation attitude? Is it possible to acquire social skills and attitudes for participation through videogames?

Any of these questions have a simple answer; this article discusses several reflection points trying to guess how this new scenario of digital gaming affects teenagers.

Keywords: Gaming, videogames, participation, social skills, social attitudes, teenagers.

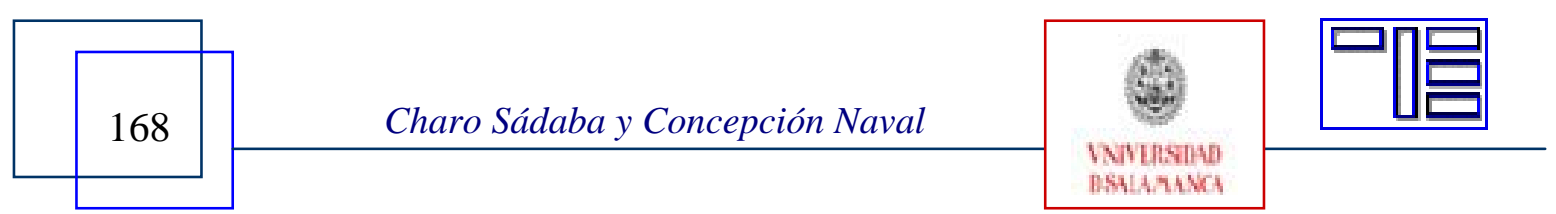




\section{UNE APPROCHE DE L'ENSEIGNEMENT VIRTUEL DE JEUX VIDÉO}

Sommaire: Est-il possible qu'une plateforme exigeant la participation constante de l'usager puisse toucher son attitude envers la participation comprise d'une manière plus large? Peut-on acquérir des attitudes et des habiletés sociales et participatives à travers les jeux vidéo?

Aucune de ces questions n'ont de réponses simples: tout au long de cet article on traite et discute plusieurs points de réflexion qui visent à leur donner une réponse dans le cadre de la réalité concrète qui entoure les adolescents.

Mots clefs: jeux, jeux vidéo, participation, habilités et attitudes sociales, adolescents.

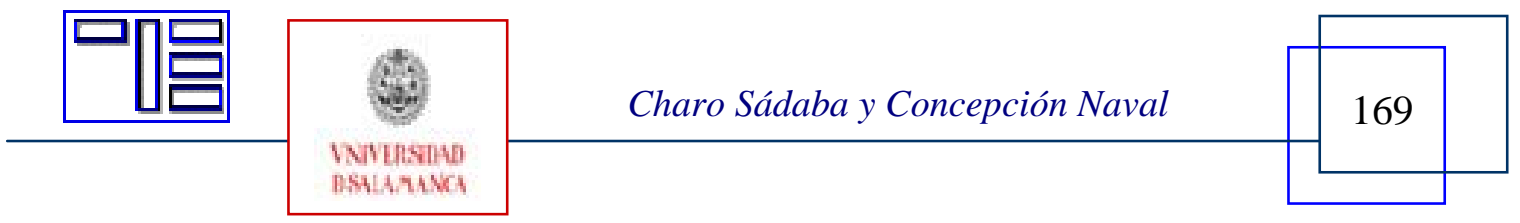




\title{
UNA APROXIMACIÓN A LA VIRTUALIDAD EDUCATIVA DE LOS VIDEOJUEGOS
}

\author{
Charo Sádaba \\ csadaba@unav.es \\ Concepción Naval \\ cnaval@unav.es \\ Universidad de Navarra
}

La capacidad de los videojuegos de captar la atención y el interés de los adolescentes es directamente proporcional al temor que han suscitado en la opinión pública en los últimos años y entre los académicos y educadores, preocupados por los posibles riesgos que su uso puede conllevar. Su alerta está justificada en parte, máxime si se piensa que esta nueva forma de ocio ocupa una gran cantidad de tiempo en la vida de los más jóvenes y que, en muchos casos, este uso se produce en solitario. El consumo de videojuegos sustituye a la televisión como "niñera catódica” y con escaso conocimiento y formación por parte de los padres.

Esta realidad que rodea a los adolescentes plantea algunas dudas entre los investigadores: ¿es posible que una plataforma que exige la continua participación del usuario pueda afectar a su actitud ante la participación entendida de un modo más amplio? ¿Se pueden adquirir habilidades sociales y participativas a través de los videojuegos?

Ninguna de estas preguntas tiene una respuesta sencilla, aunque una primera mirada a la importancia del juego en el bienestar de niños y adolescentes puede ayudar a sentar las bases para una posterior reflexión sobre la capacidad de los videojuegos para generar habilidades sociales.

\section{1.- JUEGO Y BIENESTAR}

En 2007, UNICEF publicó un amplio análisis de la vida y el bienestar de niños, niñas y adolescentes en 21 naciones económicamente avanzadas (UNICEF, 2007). Allí aparecían en cabeza como países que cuentan con los niños más felices: Holanda, Suecia, Dinamarca, Finlandia y España.

En informe tiene en cuenta seis categorías para elaborar el ranking: 1) bienestar material, 2) salud y seguridad, 3) bienestar educativo, 4) relaciones familiares y entre iguales, 5) conductas y riesgos y 6) bienestar subjetivo. Si nos ceñimos a la franja de edad

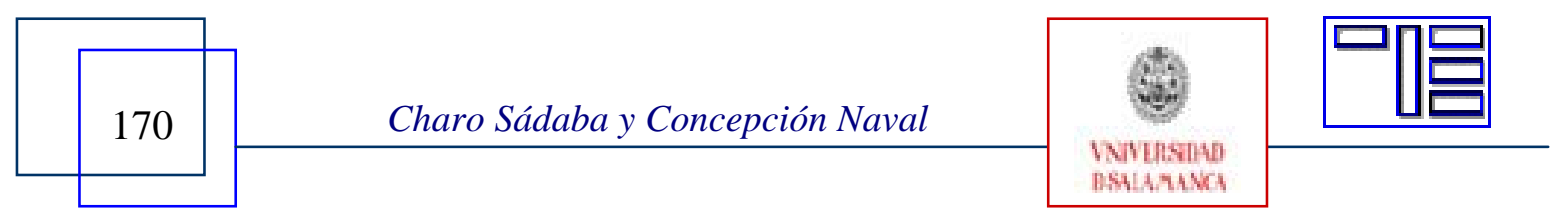


adolescente, el siguiente gráfico muestra en qué medida los integrantes de este grupo de edad se manifiestan satisfechos con su vida. Lógicamente hay diferencias en las valoraciones en función de su edad; según recoge el informe, los jóvenes de 11, 13 y 15 años más felices viven en los Países Bajos, Grecia y Finlandia.

Gráfico 1. Porcentaje de jóvenes de 11, 13 y 15 años que se valoran en la media de la escala de satisfacción vital.

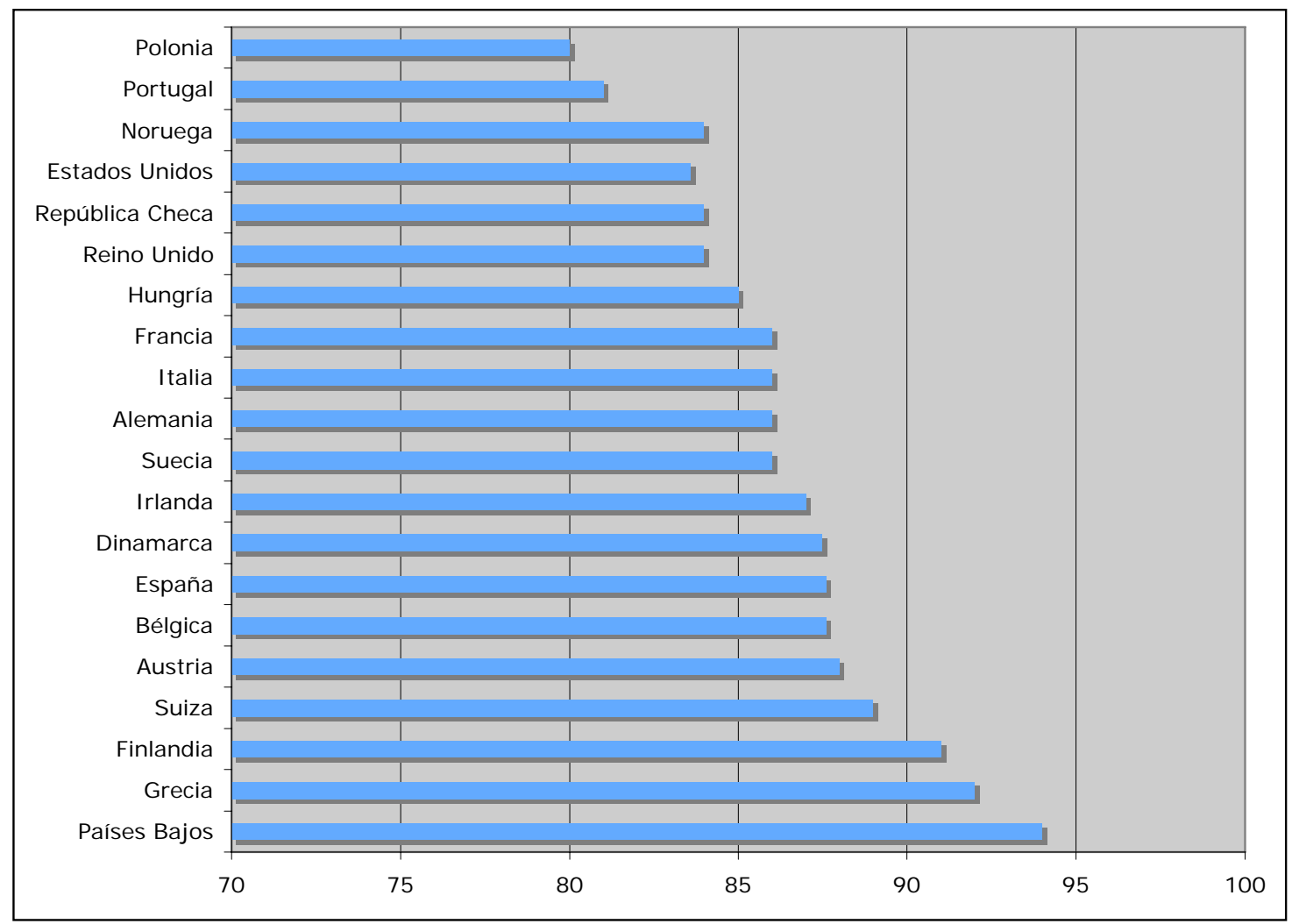

Fuente: UNICEF. Un panorama del bienestar infantil en los países ricos. 2007, p.39.

La publicación recuerda que la verdadera medida del progreso de una nación es la calidad con que atiende a sus niños: su salud y protección, su seguridad material, su educación y socialización y el modo en que se sienten queridos, valorados e integrados en las familias y sociedades en las que han nacido.

Si atendemos a la dimensión de las relaciones de los niños, los indicadores usados en el estudio citado se refieren a la estructura familiar, las relaciones familiares y las relaciones con los compañeros. La interacción de los niños con otros es un asunto de máxima importancia para el desarrollo emocional, psicológico, personal, a corto y largo plazo. Una vía ordinaria de desarrollo de esa interacción de unos niños con otros son los juegos, tanto con sus compañeros y amigos, como con sus padres u otros adultos.

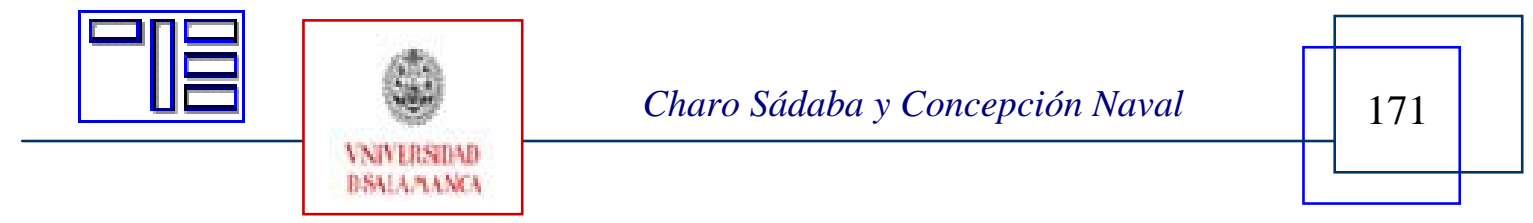


Aunque el juego carece de finalidad práctica, es decir, no trata de producir por sí mismo ninguna obra (cfr. Schwager, K; Eigler, G.; Ritzel, W., 1981, 459-460), el juego infantil se fija metas objetivas, está sujeto a reglas, se ejecuta sobre la base de una motivación infantil y su desarrollo se vive con intensidad y espontaneidad.

Como Weinert comenta, "la falta de utilidad del juego, la cuasi realidad que lleva consigo, la rápida alternativa de tensión y solución y el enfrentamiento operativo con un trozo de ambiente, lo presentan como la forma propia de actividad del niño" (Weinert, 1981, 775-776).

Se ha escrito mucho sobre el significado del juego infantil y sobre sus posibilidades formativas para el desarrollo del niño. Montesori marcó una clara línea en este sentido, que se ha seguido posteriormente.

Desde la psicología profunda (cfr. Weinert, 1981, 776 y Zullinger, 1976) se ha señalado la importancia del juego para resolver conflictos, adoptar normas sociales y una idea individual de sí mismo. "Los juegos de interpretación, de representación de roles y de reglas, son campos de experimentación en el proceso de socialización; a través de ellos el niño en la edad preescolar recibe improntas decisivas” (Weinert, p. 776).

El juego es, por tanto, medio clave para el desarrollo del niño, en diversos aspectos; también en su dimensión social. Especialmente el juego entre iguales es escuela de comportamiento y de participación social.

El juego socializado es una de las actividades más importantes en la vida de un niño. A través de él se siente feliz, aprende a imaginar, vivir y confiar en sí mismo y en los demás (Naval, Sádaba y Pérez-Alonso, en prensa).

\subsection{Los nuevos espacios del juego: los videojuegos}

Algunas investigaciones recientes ponen de manifiesto que los niños y adolescentes hoy carecen de lugares de encuentro físico que faciliten el juego tradicional. Y los dormitorios se convierten en los nuevos parques, equipados con tecnología que permite que el juego pueda darse en solitario o de modo social (Vidal y Mota, 2008). En este nuevo contexto, los videojuegos adquieren un protagonismo inusitado: en muchos casos, son la vía de juego propia de esta generación, pero también en cada vez más ocasiones, es la única vía posible muchos días a la semana, cuando estar en casa, y solo, es lo habitual.

De este modo, la interacción con otros, clave en su desarrollo emocional y psicológico, y en su bienestar global, ya no se produce sólo en el juego tradicional, sino que tiene lugar, gracias a la interactividad propia de las tecnologías de la información y de la comunicación, en el juego digital que se puede compartir con los amigos, o bien con la propia máquina que ejerce las veces de “otro”.

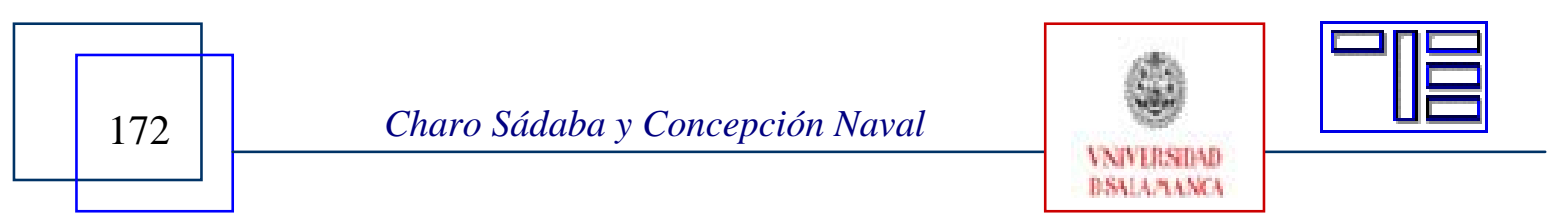


El recurso a estas plataformas de ocio interactivo consigue solventar algunas limitaciones de la vida cotidiana: no es fácil encontrar lugares donde jugar en la calle, libres de amenazas externas; además, la dispersión de los grupos humanos en las grandes ciudades hace difícil que el encuentro se pueda producir más allá de en momentos concretos y más o menos organizados del fin de semana. Esto unido a que los hogares son cada vez más pequeños, donde no siempre hay un hermano o una hermana con la que pasar el rato, ya que en muchos casos los padres tampoco están presentes en los momentos de descanso, hace que los adolescentes busquen nuevos modos y medios de entretenerse.

En este sentido es significativo el hallazgo del estudio publicado por el ente público Red.es en el año 2005, Infancia y adolescencia en la Sociedad de la Información: los datos, referidos a España, demuestran que existe una relación directa entre el hecho de que en un hogar haya niños con la probabilidad de que se disponga de un ordenador. Como puede observarse en el siguiente gráfico, con excepción de la línea telefónica fija, todas las demás tecnologías se encuentran con mayor probabilidad en una casa en la que hay niños o adolescentes.

Gráfico 2. Equipamiento tecnológico de hogares españoles sin y con hijos (niños y adolescentes)

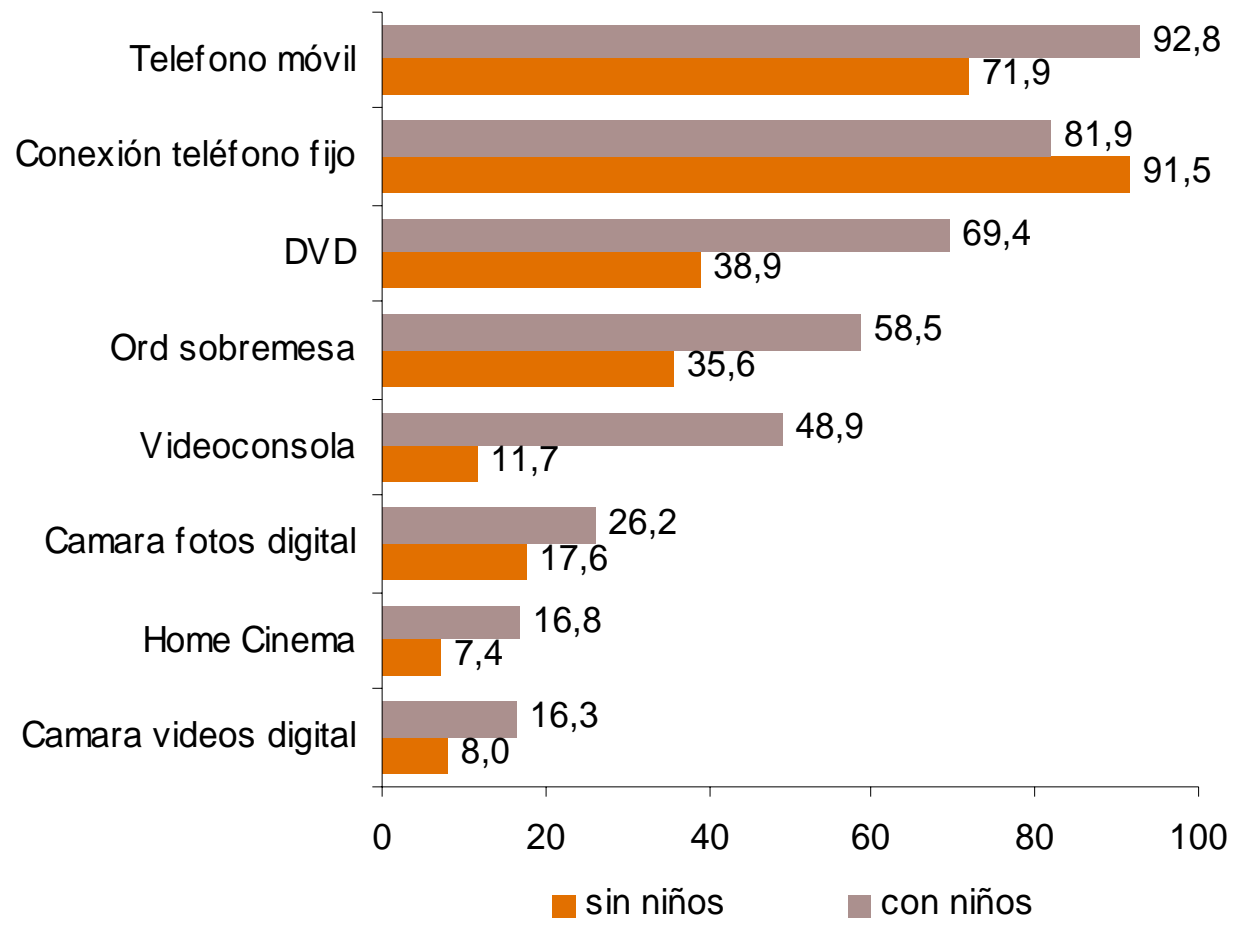

Fuente: Red.es. Infancia y adolescencia en la Sociedad de la Información. Junio 2005.

Dentro de los hogares con niños, el dormitorio de éstos se convierte, cada vez más, en la ubicación habitual de la tecnología. En este sentido, la cultura anglosajona viene estudiando desde hace años este fenómeno, conocido también como la cultura del dormito-

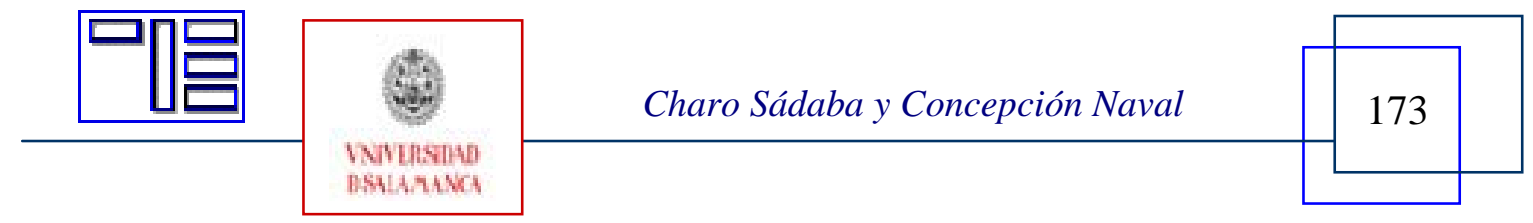


Revista Electrónica Teoría de la Educación.

Educación y Cultura en la Sociedad de la Información.

http://www.usal.es/teoriaeducacion

Vol. 9. No 3. Noviembre 2008

rio, o bedroom culture. Favorecida por el decreciente tamaño de las familias, los niños y adolescentes de hoy se encuentran, cada vez con más frecuencia, con un espacio propio dentro de su casa. La Kaiser Family Foundation, entidad norteamericana que durante años ha analizado el comportamiento de los menores en relación con las pantallas, introdujo este elemento en su análisis y, como puede apreciarse en el siguiente gráfico, es significativa la penetración de la tecnología ya no sólo en los hogares con niños, sino en sus propios dormitorios. Aunque estos datos reflejan la realidad de Estados Unidos, podemos suponer que la tendencia, tarde o temprano, tendrá vigencia también en nuestra realidad inmediata.

Gráfico 3. Equipamiento en hogares de EEUU de niños de 8-18 años

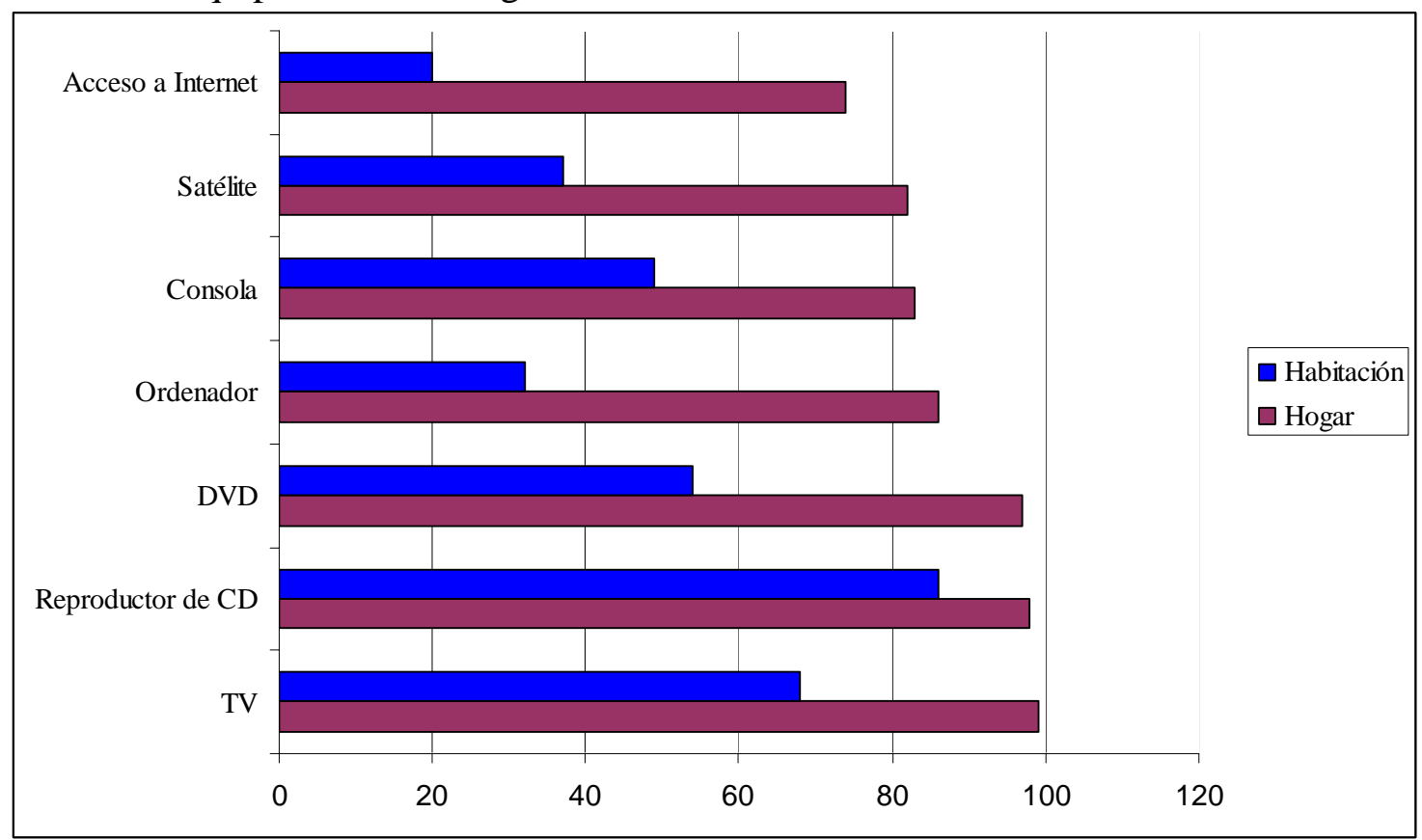

Fuente: Generación M: Media in the lives of 8- 18 Year- olds. A Kaiser Family Foundation Study. Marzo 2005

Ante la necesidad de entretenerse y la profusión de tecnología en los hogares, no es de extrañar que los videojuegos se presenten como una buena opción para hacerlo. Además, el hecho de que utilicen la interactividad les convierte en buenos candidatos para ganarse el favor de este público, ávido de participar, de crear nuevos lenguajes y códigos de comunicación (Gros, 2005; Izco, 2007; Gee, 2003).

Su precio es asequible y existen diversas plataformas que hacen que prácticamente cualquiera pueda videojugar, y la generalización de Internet de banda ancha, que permite que el juego pase de plantearse en una situación jugador-máquina, a un entorno multijugador, son otros motivos que explican su éxito entre los menores.

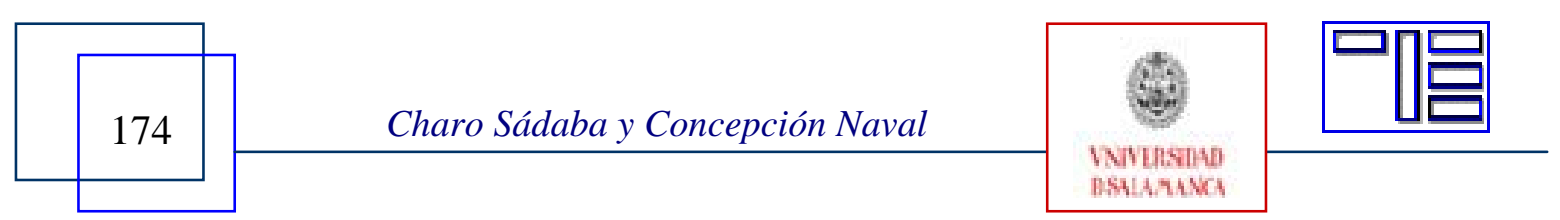


El siguiente gráfico, procedente de la Asociación Española de Distribuidores y Editores de Software de Entretenimiento (ADESE), pone de manifiesto cómo los menores, en este caso la población hasta los 19 años, supone un porcentaje importante sobre el total de jugadores en todas la plataformas: teléfonos móviles, videoconsolas y ordenadores. Este grupo de edad, hasta 19 años, supone casi el 50\% del total de los jugadores en España, como puede apreciarse.

Gráfico 4. Población española hasta 19 años con respecto al total de video-jugadores por plataformas

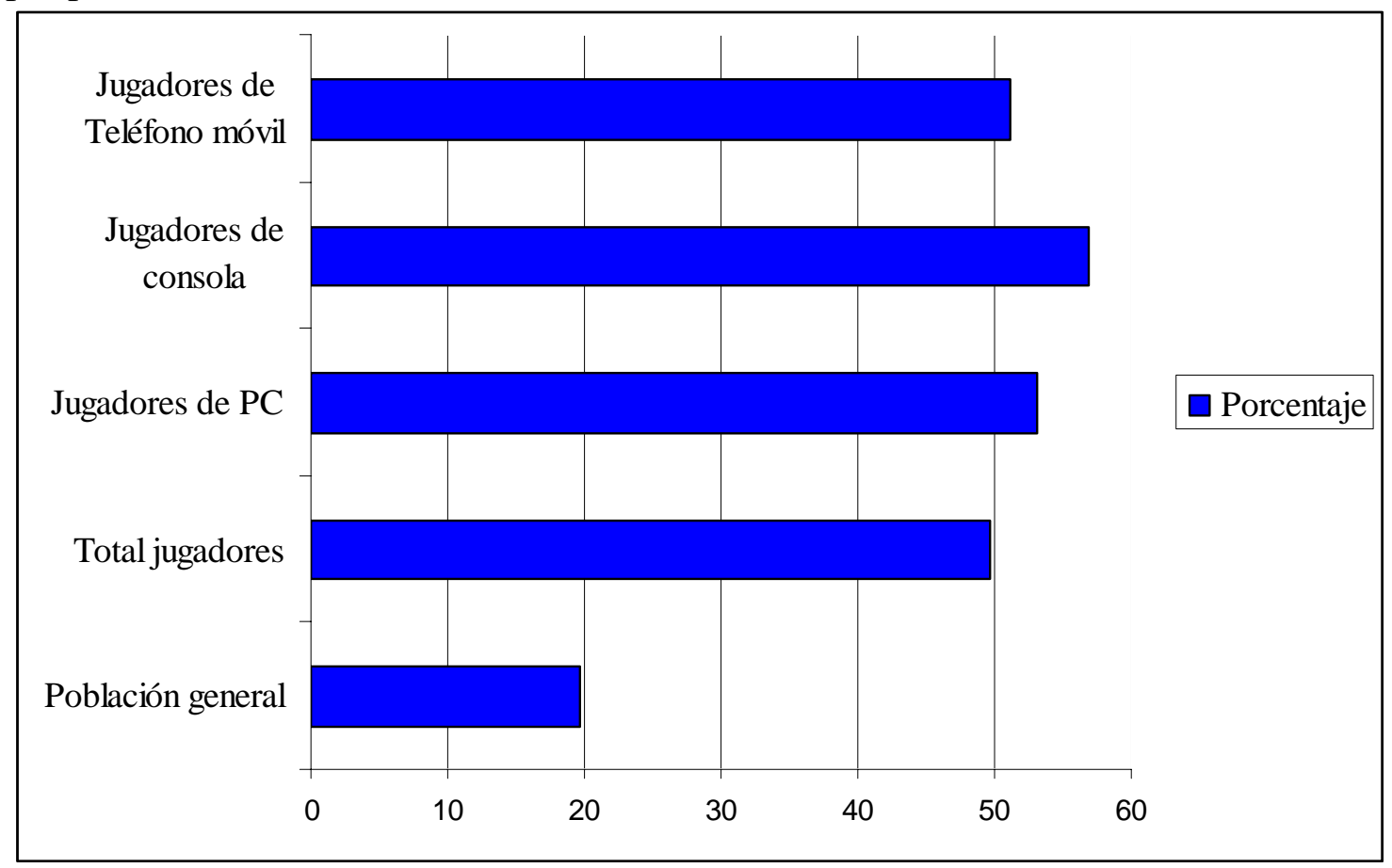

Fuente. Adese 2006

Esta omnipresencia de la tecnología, explica que al hablar de pantallas, y de videojuegos en concreto, de modo habitual se ciernan algunas sombras sobre esta visión de los juegos digitales. Así encontramos estudios donde se pone en duda que el uso de videojuegos pueda tener algún beneficio (Grossman, 2000 y Provenzo, 2001). Mientras que a otras TIC, como Internet y los móviles se les describe una virtualidad comunicativa, además de la faceta de entretenimiento, con los videojuegos no parece tan claro, ya que se apunta como predominante en ellos el aspecto de entretenimiento.

La preocupación se acrecienta cuando las plataformas supuestamente más positivas, por tener una clara finalidad comunicativa, son colonizadas por el juego como uno de los principales motivos de uso: el ordenador, el móvil y por supuesto Internet se convierten así en las plataformas de juego, como puede apreciarse en el siguiente gráfico.

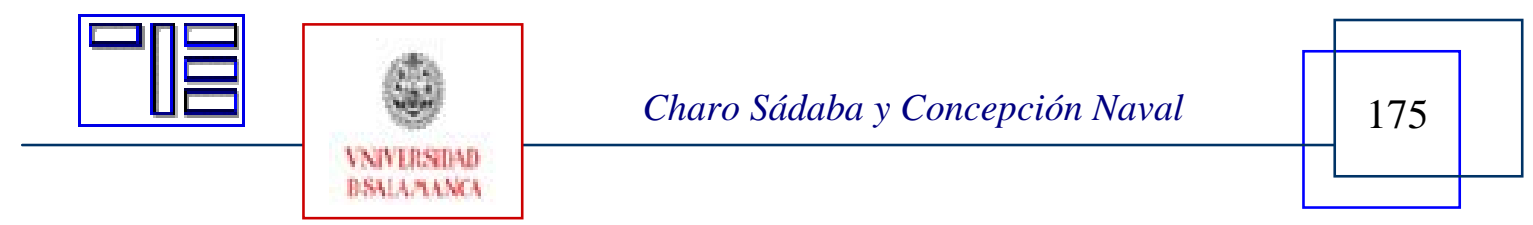


Revista Electrónica Teoría de la Educación.

Educación y Cultura en la Sociedad de la Información.

http://www.usal.es/teoriaeducacion

Vol. 9. No 3. Noviembre 2008

Gráfico 5. Frecuencia de juego con el PC, el Teléfono móvil, Videojuego. Caso español.

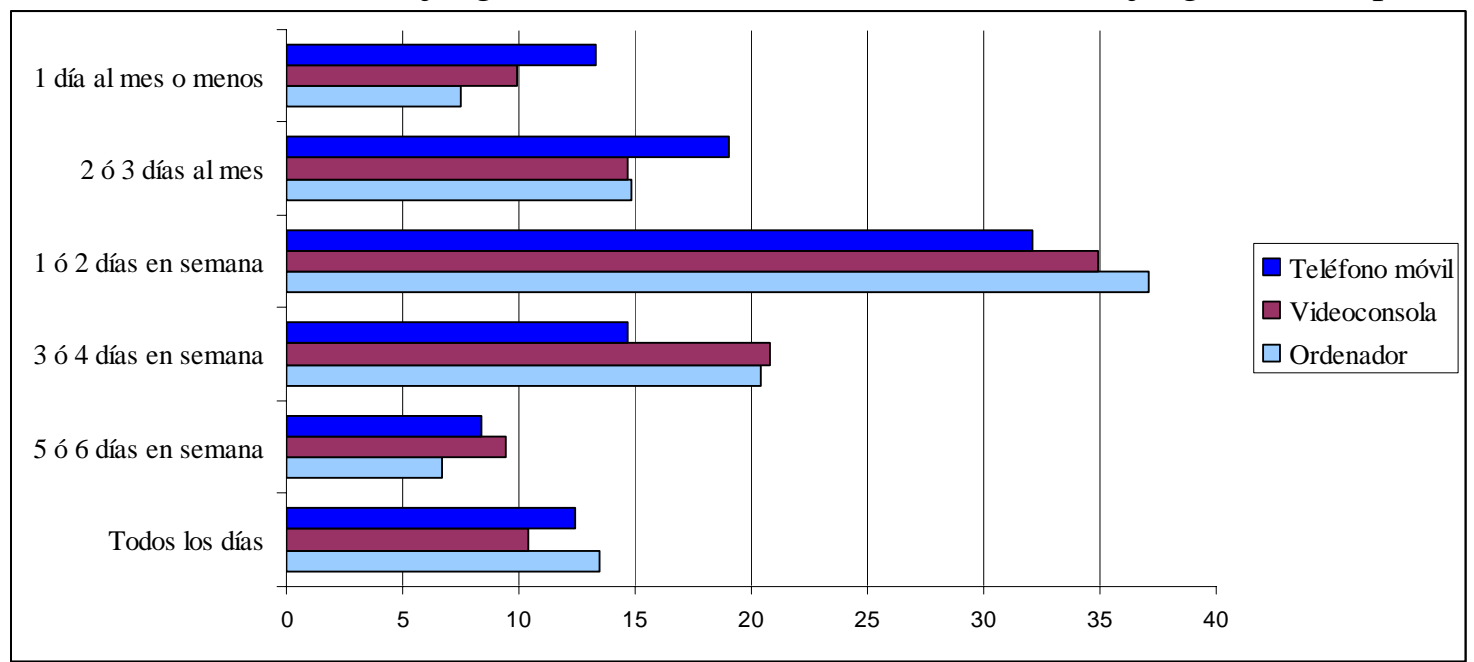

Fuente: Adese, 2006

No deja de ser paradójico que se reconozca la gran importancia del juego en la formación de niños y adolescentes, y por tanto en su satisfacción y felicidad y se adopte una postura tan radical contra los videojuegos, que suponen una nueva forma de juego que, como se verá, puede tener sus inconvenientes, pero también ofrece algunas ventajas. De hecho, y como dice Jenkins (2006, 20), no se puede perder de vista que "el significado educativo -y su potencial valor- de los videojuegos es en parte una consecuencia de su importancia creciente en la vida de los jóvenes".

Además, no conviene olvidar que los adolescentes se identifican por lo que Prensky (2001) ha dado en llamar nativos digitales: este grupo de edad ha nacido en el mundo digital, han llegado allí primero y ocupan un terreno que para los adultos es inhóspito y lleno de peligros. En este contexto, y siempre de acuerdo con Prensky, estos nativos se diferencian de los adultos, inmigrantes digitales que navegan hacia ese mundo, en su modo de comunicar (de un modo sincrónico, usando símbolos para expresar emociones); de compartir; de comprar y vender; de intercambiar, de crear, de reunirse, de coleccionar y de coordinar; de evaluar la reputación, la confianza y la credibilidad que les merece el otro; también aprenden, buscan, programan, socializan, crecen y juegan de modo diverso.

Es el conjunto de esta diversidad lo que produce una inquietud justificable en los responsables de la educación de estos nativos digitales, ya que el cambio tiende a influir en todos los aspectos de la vida, donde confluyen educación, ocio, entretenimiento y socialización. Al mismo tiempo, no hay que perder de vista que la necesidad de entretenerse es una de las características claras de los adolescentes de hoy. El entretenimiento ayuda a combatir el aburrimiento, a llenar el tiempo de ocio, es un modo de relacionarse y constituye un espacio adecuado para socializar y comunicarse. La sociedad actual eleva

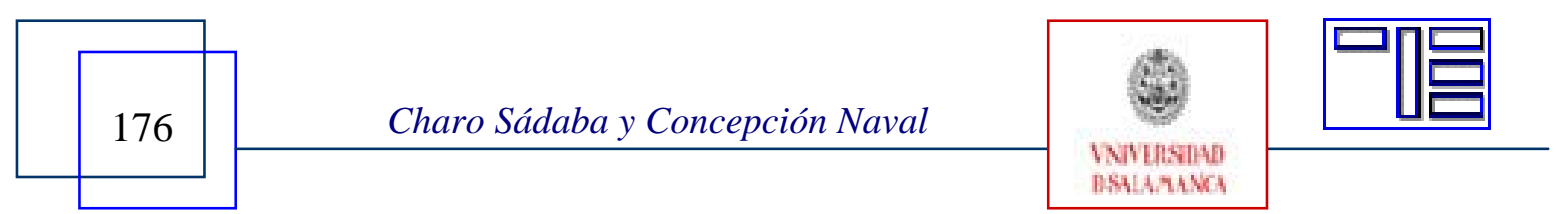


el entretenimiento prácticamente a la calidad de derecho y eso hace que los adolescentes busquen modos de conseguirlo.

Gráfico 6. Los 6 motivos principales de los menores europeos para jugar a videojuegos

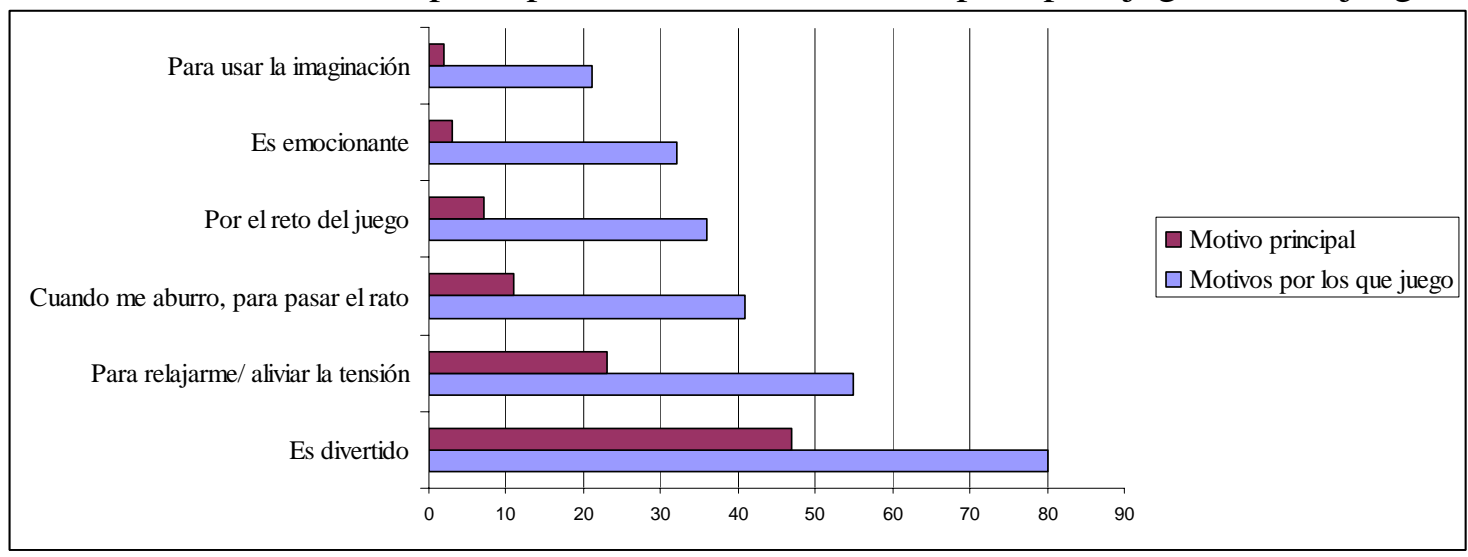

Fuente: Nielsen para la Federación Europea del Software Interactivo (ISFE). 2008.

Como se puede apreciar en el gráfico precedente, entretenerse y combatir el aburrimiento figuran como los principales motivos para videojugar.

\section{2.- VIDEOJUEGOS Y HABILIDADES SOCIALES}

Que los videojuegos pueden ayudar a la adquisición de contenidos y de algunas competencias clave es una idea constante en las últimas investigaciones sobre el tema (Gros, 2008; Bernat, 2008). Existen cada vez más experiencias sobre cómo un juego puede ayudar a aprender un idioma, historia, cálculo, o ayudar a comprender la integración. Sin embargo, el tema que nos ocupa en estas páginas tiene que ver con la capacidad de estas plataformas para suscitar habilidades y actitudes sociales, principalmente habilidades participativas y comunicativas. Estas habilidades ayudan a incrementar el compromiso cívico. Entre ellas, podemos destacar:

a. Las habilidades de comunicación

b. Las habilidades de negociación

c. La capacidad de resolución de problemas y conflictos

d. La iniciativa personal

e. El trabajo en equipo

¿En qué medida los videojuegos pueden ayudar a desarrollar estas habilidades? Parece claro que el uso razonable de estas plataformas de ocio interactivo puede fomentar la iniciativa personal, y el trabajo en equipo.

En la medida en que el juego, al tener lugar sobre una plataforma interactiva, no sucede sin la intervención activa del usuario, la iniciativa es clave y esencial para que el juego tenga lugar. Además, esa iniciativa, respondiendo a los objetivos previos del juego, de-

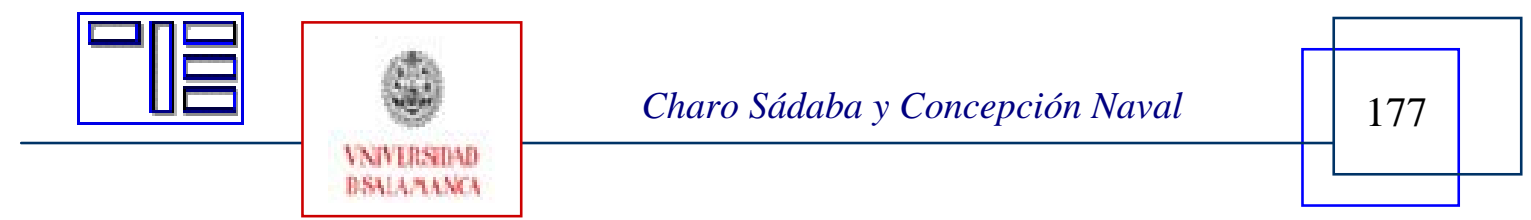


be tener en cuenta sus límites y sus fortalezas, por lo que se ayuda a orientar, a focalizar, este esfuerzo personal para conseguir una meta.

En la medida en que los juegos en red se hacen más populares, el trabajo colaborativo aparece como necesario y, por lo tanto, puede suscitar o fomentar la habilidad de trabajar en equipo. De nuevo, este trabajo deberá estar al servicio de un objetivo común compartido, lograr la meta del juego, y tener en cuenta las normas propias del espacio de ocio.

La posibilidad de que los videojuegos ayuden a fomentar la capacidad de resolución de problemas y conflictos está emergiendo en los últimos años como una potencialidad a descubrir. El hecho de que estos juegos digitales se planteen como una sucesión de retos que hay que resolver de un modo estratégico, puede ayudar en este sentido.

En menor medida, aunque en cierta manera impregna el uso de las tecnologías de la información y de la comunicación, parece que los videojuegos podrían ayudar también al desarrollo de habilidades comunicativas. Sin embargo, aquí la comunicación tiene un carácter instrumental, en el sentido de que su fin es lograr la meta planteada, o facilitar el trabajo en equipo.

Las habilidades de negociación aparecen como menos probables, aunque no hay que despreciar que algunos videojuegos en particular, basen su estrategia precisamente en la negociación: es el caso de uno de los juegos más populares en España, Animal Crossing: avanzar en el juego requiere negociar con los demás habitantes de los pueblos, intercambiar productos para sobrevivir, etc.

Los adolescentes son grandes usuarios de las plataformas de juego interactivo, como se ha apuntado. Éstas se caracterizan por demandar la participación activa del usuario que no es un mero espectador (como en el caso del cine), sino que puede determinar el curso de la trama del juego con sus acciones. Como afirman Gros (2005) e Izco (2007), esta interactividad de las plataformas las convierte en especialmente deseables y atractivas para este grupo de edad. Como afirma Jenkins (2006, 20), es precisamente a consecuencia de este uso, cuando hay que pensar en el posible valor y potencial educativo de estas plataformas. Por lo tanto, hay que aceptar que los jóvenes ya han adoptado esta realidad, y que sólo cabe aceptarla e integrarla en la educación.

La exposición a la violencia, y la propia participación en ella precisamente por el carácter interactivo del medio, es uno de los puntos de mayor preocupación cuando se habla de videojuegos. Sin embargo, y pese a reconocer el valor de aportaciones como la de Grossman, para quien los adolescentes son brutalizados por una sobreexposición a la violencia a una edad en la que no pueden siempre distinguir entre representación y realidad, aparecen opiniones más recientes, como la de Jenkins, que merece ser tenida en cuenta. Según este autor (2006, 23), la posición que narra Grossman sólo es posible si se asume que los jugadores no son capaces de pensamiento racional, si se ignoran las

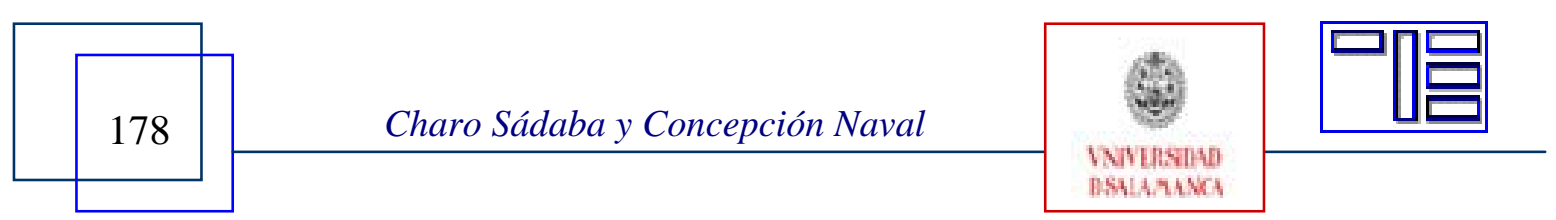


diferencias críticas en cómo y por qué la gente juega y se obvia cualquier contexto educativo o cultural significativo.

Hay que tener en cuenta que cuando los jóvenes juegan, "no se sientan frente a su consola a aprender una lección. Su atención está mucho más fragmentada; sus metas son mucho más personales; y no van a ser evaluados por lo que aprenden” (Jenkins 2006, 22). Todo esto obliga a repensar los posibles efectos de la violencia, que no pueden ser evaluados sin tener en cuenta el contexto y la actitud del jugador ante la pantalla.

Además, frente a los videojuegos de cartucho, con unas pocas horas de juego posible, y con unos límites claros, la preferencia de los adolescentes por el juego en red es creciente: en este caso, la historia tiene una duración infinita, en la medida en que son los propios usuarios quienes alimentan el juego con nuevos personajes, tramas y objetivos. Participar en estas plataformas les obliga a pensar en la comunidad de videojugadores y a diseñar estrategias que animen a otros a la participación. De hecho es uno de los motivos para jugar. De acuerdo con el proyecto de investigación "Generaciones Interactivas en Iberoamérica: retos educativos y sociales" -que ha encuestado entre marzo y junio de 2008 a más de 80.000 niños y niñas entre 6 y 18 años en Argentina, Brasil, Chile, Colombia, México, Perú y Venezuela- este grupo de edad prefiere jugar con otros, como puede apreciarse en el siguiente gráfico:

Gráfico 7. ¿Estás de acuerdo con las siguientes frases?

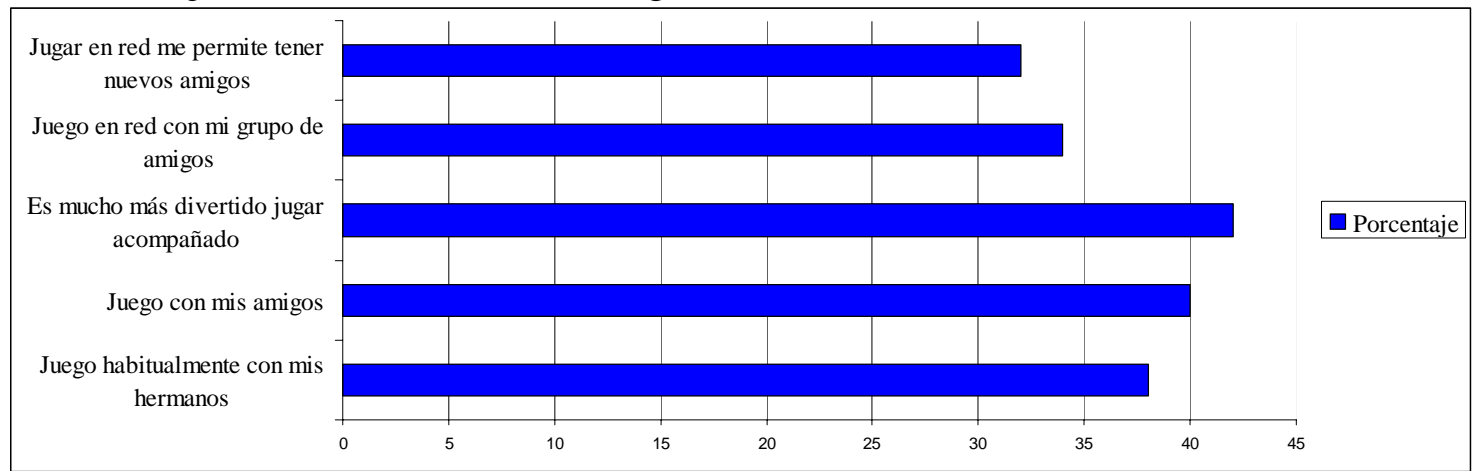

Fuente: Generaciones Interactivas. 2007. Datos referidos a menores de Argentina, Chile, Colombia, Brasil, Perú, México y Venezuela.

Relacionando este punto con el anterior, Wright (2002) afirma que en los videojuegos violentos, hay dos juegos en marcha: "uno, el combate explícito en la pantalla, el otro, la cooperación y la camaradería implícita entre los jugadores (2006, 25). A este respecto es interesante también otro dato: no son muchos los padres o madres que se aventuran a participar con sus hijos en estos nuevos escenarios de juego. Sin embargo, los motivos que llevan a algunos a hacerlo parecen relevantes: "mi hijo/s me pide que juegue con él/ella”, es la razón más importante para muchos padres europeos, tal y como pone de

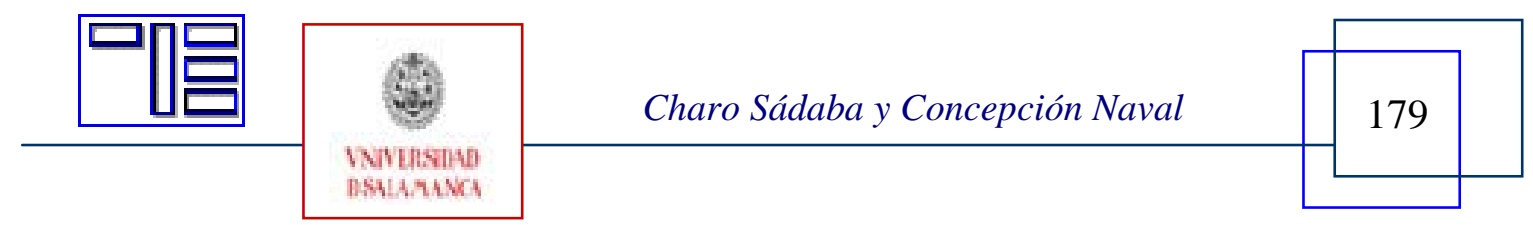


Revista Electrónica Teoría de la Educación.

Educación y Cultura en la Sociedad de la Información.

http://www.usal.es/teoriaeducacion

Vol. 9. No 3. Noviembre 2008

manifiesto el siguiente gráfico, referido a los motivos por los que los padres europeos juegan con sus hijos.

Gráfico 8. ¿Por qué juega con su hijo/s? ¿Y cuál es el motivo principal? Datos Europeos

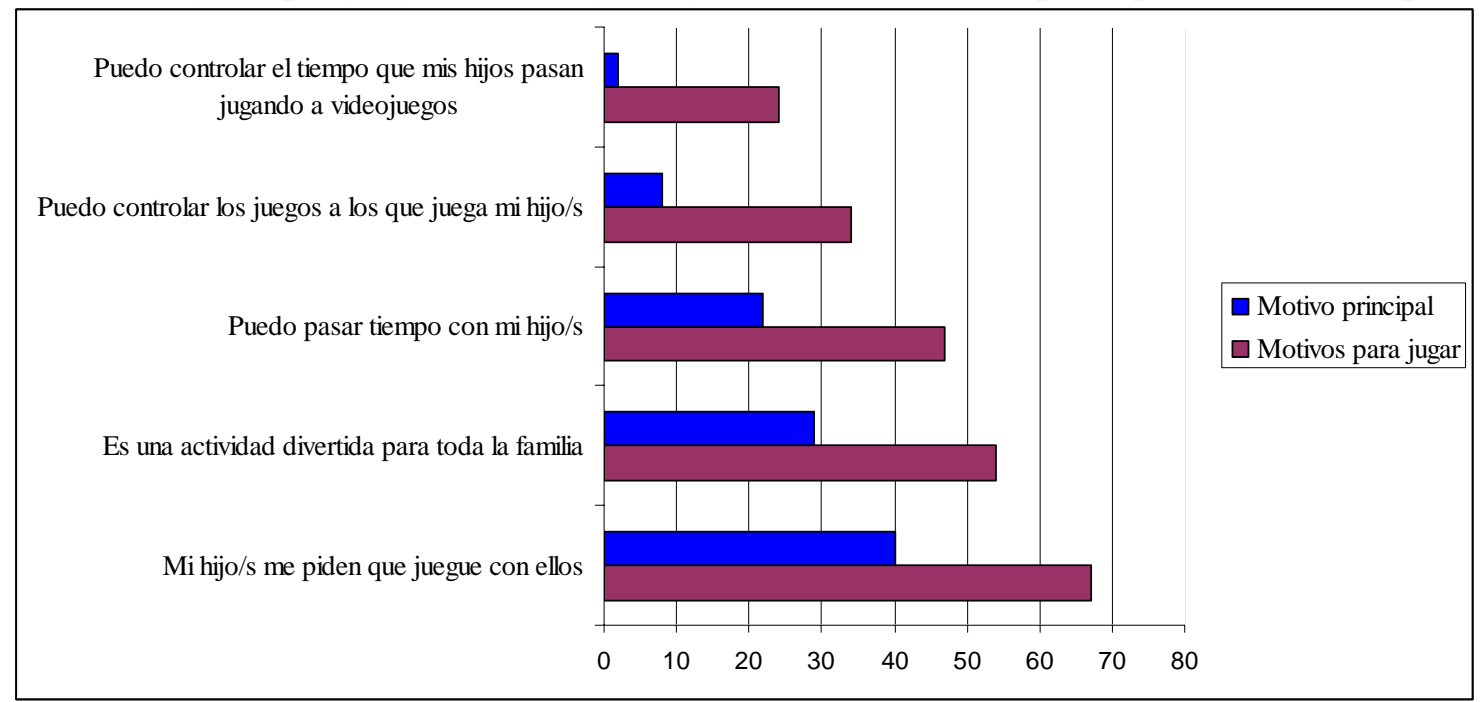

Fuente: Nielsen para la Federación Europea del Software Interactivo (ISFE). 2008.

Parece que en muchos casos el juego solitario con la plataforma tecnológica es obligado para el adolescente: no tiene con quién jugar y, sin embargo, preferiría hacerlo con otro. Es cierto, como se ha visto, que el principal motivo por el que los menores juegan es lúdico: entretenerse. Esto hace que de entrada sea difícil establecer una relación directa entre el uso de los videojuegos y la posible participación en asuntos no relacionados con los juegos. Sin embargo, también se aprecia que los videojuegos pueden tener un potencial de sociabilidad relevante. Junto con esto, es interesante la visión de Gee, según el cual "los videojugadores son activos solucionadores de problemas, que ven los errores como oportunidades para aprender y reflexionar, y que están constantemente buscando nuevas y mejores soluciones a los obstáculos y retos” (Gee, 2003, 23).

Este carácter lúdico se manifiesta también en que, aunque existen en el mercado videojuegos educativos, o que de un modo explícito buscan fomentar determinadas actitudes positivas en el jugador, las preferencias de los jóvenes se rigen por criterios comerciales.

Como se puede apreciar en la tabla 1, los dos juegos más populares en España durante el año 2007 han sido dos títulos que precisamente proporcionan un modo de mantener y adquirir ciertas habilidades mentales. Los deportes, y más en concreto el fútbol, es un contenido de éxito en estas plataformas, que ocupan los puestos 3, 8 y 9. Los juegos con un claro componente de aventura como Pokemon Diamante y New Super Mario Bros también ocupan posiciones importantes. Y la aparición de Animal Crossing: Wild World, que requiere un juego colaborativo, hace patente el carácter social que pueden tener también los videojuegos. Todos los títulos de la lista, con excepción de WWE

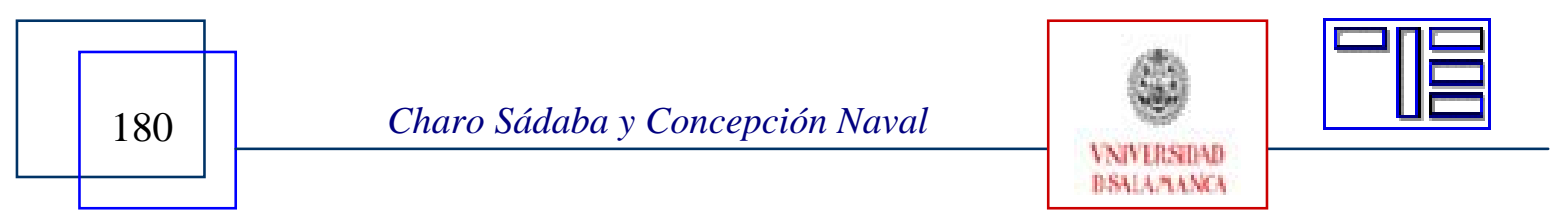


Smackdown! tienen una calificación según el sistema PEGI como aptos para mayores de 3 años, lo que pone de manifiesto que los menores son destinatarios clave de este mercado

Tabla 1. Videojuegos España: 10 títulos más vendidos en 2007

\begin{tabular}{|l|l|l|l|l|}
\hline Plataforma & Título & $\begin{array}{l}\text { Distribui- } \\
\text { dor }\end{array}$ & Género & Edad \\
\hline 1. DS & Mas Brain Training & Nintendo & Estrategia & $3+$ \\
\hline 2. DS & Brain Training del Dr. Kawashima & Nintendo & Estrategia & $3+$ \\
\hline 3. PS2 & Pro Evolution Soccer 2008 & Konami & Deportes & $3+$ \\
\hline 4. WII & WII Play & Nintendo & Otros & $3+$ \\
\hline 5. DS & Pokemon Diamante & Nintendo & Aventura Graf./RPG & $3+$ \\
\hline 6. DS & New Super Mario Bros & Nintendo & Plataforma & $3+$ \\
\hline 7. PS2 & WWE Smackdown! Vs Raw 2008 & THQ & Acción/ Combate & $16+$ \\
\hline 8. PS2 & Pro Evolution Soccer 6 Platin & Konami & Deportes & $3+$ \\
\hline 9. PS3 & Pro Evolution Soccer 2008 & Konami & Deportes & $3+$ \\
\hline 10. DS & Animal Crossing: Wild World & Nintendo & Aventura Graf./RPG & $3+$ \\
\hline
\end{tabular}

Fuente: Adese; 2008.

\section{3.- REFLEXIONES FINALES}

Resulta necesario conocer las motivaciones que llevan a los jóvenes al uso de estas plataformas de ocio interactivo. La necesidad del otro, la búsqueda común del entretenimiento, la necesaria cooperación con los otros para conseguir metas comunes en muchas plataformas, auguran al menos unas disposiciones que luego podrán ser reforzadas y empleadas en otros ámbitos.

Todo esto lleva consigo, como afirma Buckingham (2003), la necesidad de repensar la educación sobre los nuevos medios, que él denomina un "nuevo paradigma”, según el cual hay que tratar de proteger menos a los niños y adolescentes de los efectos perniciosos de los medios, y en cambio promover más la comprensión y la participación en la cultura de los medios. Si consideramos que el juego encierra una dimensión educativa, el hecho de que dicho juego suceda a través de la tecnología añade un elemento tecnológico, pero no resta fuerza al valor de esta actividad.

\section{4.- BIBLIOGRAFÍA}

ADESE. (2006). Estudio de Hábitos y Consumo de Videojuegos. GFK Group. Disponible en http://www.adese.es/web/informes.asp

BERNAT CUELLO, A. (2008). "La construcción de conocimientos y la adquisición de competencias mediante el uso de los videojuegos", en GROS, B. (coord.). Videojuegos y aprendizaj. Graó, Barcelona.

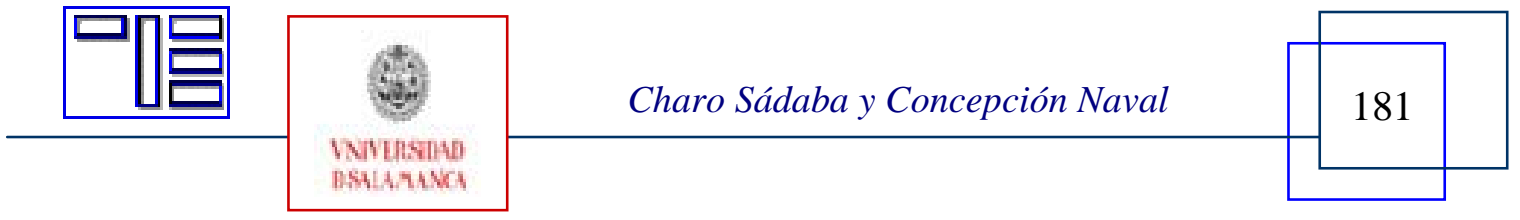


BRINGUE, X. y SÁDABA, Ch. (coord.) (2008). La generación interactiva en Iberoamérica. Niños y adolescentes frente a las pantallas. Colección Fundación Telefónica. Ariel, Barcelona.

BUCKINGHAM, D. (2003). Media education: Literacy, learning, and contemporary culture. Cambridge, Polity.

GEE, J. (2003). What Video Games Have to Teach Us About Learning and Literacy. New York, Palgrave.

GROS, B. (2005): Pantallas y juegos: de la observación de modelos a la participación, en NAVAL, C. y SÁDABA, Ch. (coords.): Jóvenes y medios de comunicación. Revista de Estudios de Juventud, 68, 61-71.

GROS, B. (coord.). (2008). Videojuegos y aprendizaje. Graó, Barcelona.

GROSSMAN, D. (2000). Teaching kids to kill. Phi Kappa Phi, “National Forum”. Disponible en http://www.killology.org/article_teachingkid.htm, accedido en junio de 2008.

IZCO, E. (2007). Los adolescentes en la planificación de medios. Segmentación y conocimiento del Target. Tesis defendida en la Facultad de Comunicación de la Universidad de Navarra. Directores: Idoia Portilla y Francisco Javier Pérez Latre. Leída: 25 de enero de 2007.

JENKINS, H. (2006). The War Between Effects and Meaning: Rethinking the Video Game Violence Debate, en BUCKINGHAM, D. y WILLETT, R. (2006) Digital Generations: children, young people, and new media, New Jersey, Lawrence Erlbaum Associates.

NAVAL, C., SADABA, Ch. y ALONSO-JETA, P. (en prensa). Relaciones de pares, tecnologías de la comunicación y educación ciudadana. México.

NIELSEN (2008). Usuarios de videojuegos en Europa. Informe para la Federación Europea del Software Interactivo (ISFE). Disponible en http://www.adese.es/web/informes.asp, accedido en agosto de 2008.

PRENSKY, M. (2001). Digital Game-based Learning. New York, McGraw Hill.

PROVENZO, E. (2001). Childen and hypereality: The loss of the real in contemporary childhood and adolescence. Paper presentado en la conferencia en el Cultural Policy Center de la Universidad de Chicago. Disponible en http://culturalpolicy.uchicago.edu/conf2001/papers/provenzo.html, accedido en junio de 2008.

RED.ES (2005). Infancia y adolescencia en la Sociedad de la Información. Madrid, Observatorio de las Telecomunicaciones y de la Sociedad de la Información.

ROBERTS, D. F.; FOEHR, U.G.; \& RIDEOUT, V. (2005, marzo). Generación M: Media in the lives of 8- 18 Year- olds. Menlo Park, California. Kaiser Family Foundation. Disponible en http://www.kff.org/entmedia/upload/Generation-M-

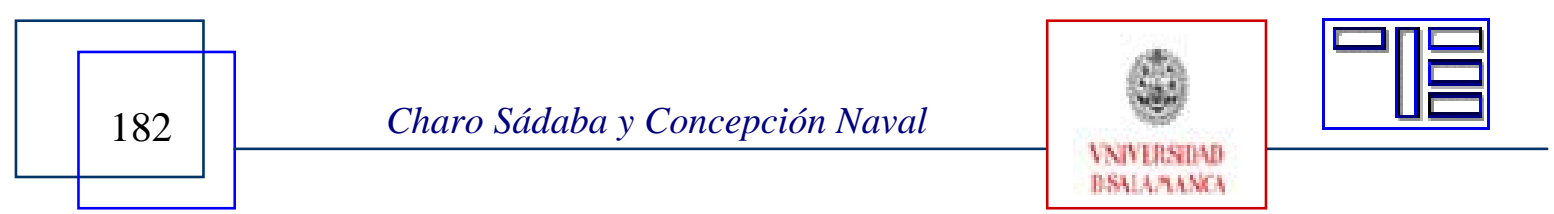


Media-in-the-Lives-of-8-18-Year-olds-Report.pdf, accedido en septiembre de 2008.

SCHWAGER, K; EIGLER, G.; y RITZEL, W. (1981). Voz Metodología, en SPECK, J. y WEHLE, G., Conceptos fundamentales de Pedagogía. Barcelona, Herder.

UNICEF (2007). Pobreza infantil en perspectiva. Un panorama del bienestar infantil en los países ricos. Un amplio análisis de la vida y el bienestar de niños, niñas y adolescentes en las naciones económicamente avanzadas. Florencia, Centro de Investigaciones Innocenti de UNICEF.

VIDAL, F. y MOTA, R. (2008). Encuesta de la infancia en España. Fundación SM, Madrid.

WEINERT, F. (1981). Voz Psicología del desarrollo, en SPECK, J. y WEHLE, G., Conceptos fundamentales de Pedagogía. Barcelona, Herder.

WRIGHT, T. (2002). Creative players in action in FPS online videogames: Playing counter-strike. Game Studies. Disponible http://www.gamestudies.org/2002/wright/, accedido en junio de 2008.

ZULLINGER, H. (1976). Evolución psicológica del niño. Barcelona, Herder.

Para citar la presente editorial puede utilizar la siguiente referencia:

SÁDABA, Charo y NAVAL, Concepción (2008). Una aproximación a la vietualidad educativa de los videojuegos. En SÁNCHEZ i PERIS, Francesc J. (Coord.) Videojuegos: una herramienta educativa del "homo digitalis" [monográfico en línea]. Revista Electrónica Teoría de la Educación: Educación y Cultura en la Sociedad de la Información. Vol. 9, nº 3. Universidad de Salamanca [Fecha de consulta: $\mathrm{dd} / \mathrm{mm} / \mathrm{aaaa}$ ]. http://www.usal.es/ teoriaeducacion/rev_numero_09_03/n9_03_sadaba_naval.pdf ISSN: 1138-9737

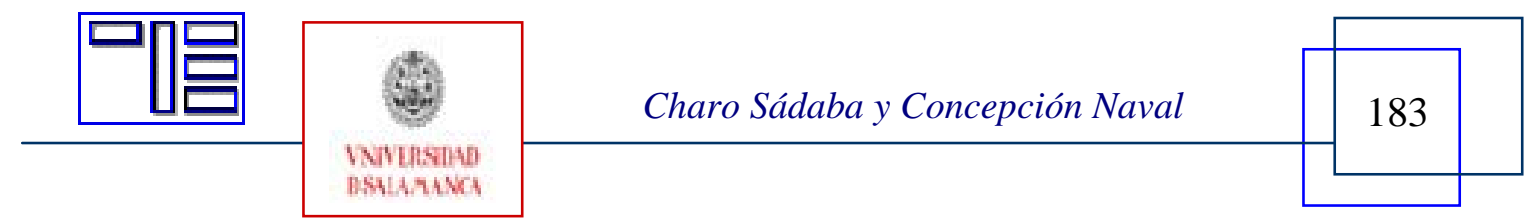

